\title{
Palm pattern recognition using scale invariant feature transform
}

\section{Kasiselvanathan*, V. Sangeetha and A. Kalaiselvi}

\author{
Electronics and Communication Engineering, \\ Sri Ramakrishna Engineering College, \\ Coimbatore, Tamil Nadu, 641 022, India \\ Email: kasiselvanathan@gmail.com \\ Email: sangeetha.v@srec.ac.in \\ Email: kalaiselvi.a@srec.ac.in \\ *Corresponding author
}

\begin{abstract}
In this research paper, we propose an efficient scale invariant feature transform (SIFT) for palm pattern recognition. A fingerprint recognition which is efficient for individual authentication based on fingerprint pattern. This method leads to fraudulent because it could be extracted easily from individuals. The SIFT method based on feature detection overcomes the above problem and is a combination of fast key point detector and visual descriptor. Using SIFT method contactless palm pattern images can be acquired, matched, recognised, authenticated and their matching performance are simulated using OpenCV. The experimental results show that SIFT method provides significantly fast and improved performance than the conventional methods like oriented FAST and rotated BRIEF (ORB).
\end{abstract}

Keywords: image matching; biometrics; palm pattern; feature detection; scale invariant feature transform; SIFT; ORB.

Reference to this paper should be made as follows: Kasiselvanathan, M., Sangeetha, V. and Kalaiselvi, A. (2020) 'Palm pattern recognition using scale invariant feature transform', Int. J. Intelligence and Sustainable Computing, Vol. 1, No. 1, pp.44-52.

Biographical notes: $\mathrm{M}$. Kasiselvanathan is pursuing his $\mathrm{PhD}$ and received his Master's in VLSI Design. He is now an Assistant Professor at the Department of Electronics and Communication Engineering of Sri Ramakrishna Engineering College, Coimbatore, Tamilnadu, India. His main research areas are wireless communication, signal and image processing, and low power VLSI.

V. Sangeetha completed her Post-graduate in VLSI Design from the Anna University. She is now an Assistant Professor at the Department of Electronics and Communication Engineering of Sri Ramakrishna Engineering College, Coimbatore, Tamilnadu, India. Her area of interests includes image processing, analogue VLSI and mixed signal VLSI.

A. Kalaiselvi completed her $\mathrm{PhD}$ in Image Processing and obtained post graduate in Applied Electronics. She is now an Assistant Professor at the Department of Electronics and Communication Engineering of Sri Ramakrishna Engineering College, Coimbatore, Tamilnadu, India. Her area of specialisations includes image processing, wireless communication and embedded systems. 


\section{Introduction}

The contactless palm print and palm vein recognition system uses a hand sensor that could capture the palm print and palm vein image using low resolution web camera. The local ridge enhancement (LRE) method removes illumination error while keeping good contrast between the print pattern and the background image (Michael et al., 2010). Multi-modal biometric recognition systems have greater advantages that provide good anti-spoofing abilities. The system uses Contourlet transform to analyse the features present in palm print and palm vein images (Gaikwad and Narote, 2014). Feature detection is used to compute the image information and make a local decision at every image point to check whether there is an image feature of the given type existing in that point. This feature detection technique provides robust to image transformations (Moghaddam et al., 2001; Shan, 2010). Several variants and extension of scale invariant feature transform (SIFT) methods have been introduced to improve its computational complexity (Güzel, 2015; Chiu et al., 2013; Ke and Sukthankar, 2004). Speed up robust feature (SURF) technique is an approximation of SIFT method to perform faster than SIFT techniques in detected points (Bay et al., 2008).

A novel palm vein recognition system is introduced which is based on the enhanced local gabor binary patterns histogram sequence (ELGBPHS) algorithm. The ELGBPHS algorithm is a well established face recognition algorithm for palm vein recognition (Fischer et al., 2012). The performance of different image matching techniques is compared against different kinds of transformations and deformations. A robust descriptor based on BRIEF, called RBRIEF is proposed to scale and in-plane rotation transformations (Huang et al., 2013). Palm dorsa subcutaneous vein pattern (PDSVP) has a reliable and promising physiological biometric feature used for biometrics related research. A near infrared (NIR) imaging of PDSVP system has been introduced for data acquisition, PDSVP extraction (Joardar et al., 2016). Finger or palm dorsal surface is used for fingerprints during border crossings or during real life activities. The lowest finger knuckle patterns formed on joints between the metacarpal and proximal phalanx bones for the automated personal identification have been discussed (Kumar and $\mathrm{Xu}$, 2016).

In Mahesh and Subramanyan (2012), many feature based registration methods were discussed. It also gives SIFT algorithm for image registration to detect the features such as points, lines, corners and objects. Face recognition technique is used to detect the face in the images so that it could be used in many applications especially in the security (Wong et al., 2011). SIFT algorithm is invariant to rotation, scale, brightness change. SIFT feature can be used to compute massive feature data and to combine with other types of feature matching. In Jinbo et al. (2012), the circular region is divided into four concentric circles with a radius of two pixels, and every sub-region is divided into ten directions to generate a forty dimensional feature descriptor. In Shuzhen et al. (2010), proposed method using circular region computes every sub-region's gray cumulative value and difference value between every sub-region to generate descriptor. In Luan and Dalong (2012), a proposed method to generate descriptor based on a circular region divides the circular into eight fan-shaped area of equal area, and every sub-region has eight orientations, forming a 64 dimensional feature descriptor. Jia et al. (2013) propose a method which is used to divide the rectangular neighbourhood into square rings to take place of squares. The vein pattern based on biometric identification technique has been 
proposed to analyse the pattern of blood vessels visible under skin of the person (Srinithi and Gopalakrishnan, 2017). Palm vein recognition relying on high dynamic range (HDR) imaging for better recognition performance than a baseline system relying on singleexposure acquisitions. The experimental results improve the performance of the proposed method when discriminative features are extracted from HDR contents, with respect to the use of single-exposure images (Piciucco et al., 2018). In Vamsikrishna et al., (2016), a computer vision assisted contactless methodology is proposed to facilitate the palm pattern and finger rehabilitation. Experimental results show that the proposed technique is better than the isolated gesture recognition. The SURF, oriented SIFT and rotated BRIEF (ORB) methods are discussed in Karami et al. (2015). The SIFT feature detection used to establish a space mapping model based on BP neural network which finds the number of mismatching points around the coordinate (Liao and Wang, 2015).

\section{Block diagram of proposed method}

Conventional authentications such as passwords, fingerprint authentication, smart cards are used for security purposes. The biometric security techniques have some advantages include physiological (face, iris, fingerprint, shape of hand) and behavioural (sign, handwriting) identifications. Among these identification techniques, fingerprint recognition provides better security for individual authentication based on fingerprint pattern. But due to wounds, oil patches, dirt and age disorders, the fingerprint technique has some negative effect. This technique leads to fraudulent because it may be extracted easily from individuals. To avoid such fraudulent we propose a novel palm pattern identification system based on feature detection. The schematic diagram of overview of proposed approach is shown in Figure 1. The process of the block diagram is explained below.

\subsection{Segmentation of hand shape}

Segmentation of coloured images produces negative effects as stated in previous related work. In our proposed approach, before segmentation BGR images are converted into greyscale images. Palm pattern ROI can be extracted which includes hand shape segmentation. And poor images may occur due to lighting conditions. To avoid such a noisy region, SIFT algorithm is used to segment the hand shape.

\subsection{Extraction and normalisation of $R O I$}

A midpoint of palm pattern is taken as reference to extract a ROI from palm pattern image. Scaling and rotation correction of palm pattern images can be normalised using a bilinear gray value differential method. The square of the normalisation defines palm pattern ROI. 
Figure 1 Schematic diagram of overview of proposed approach (see online version for colours)

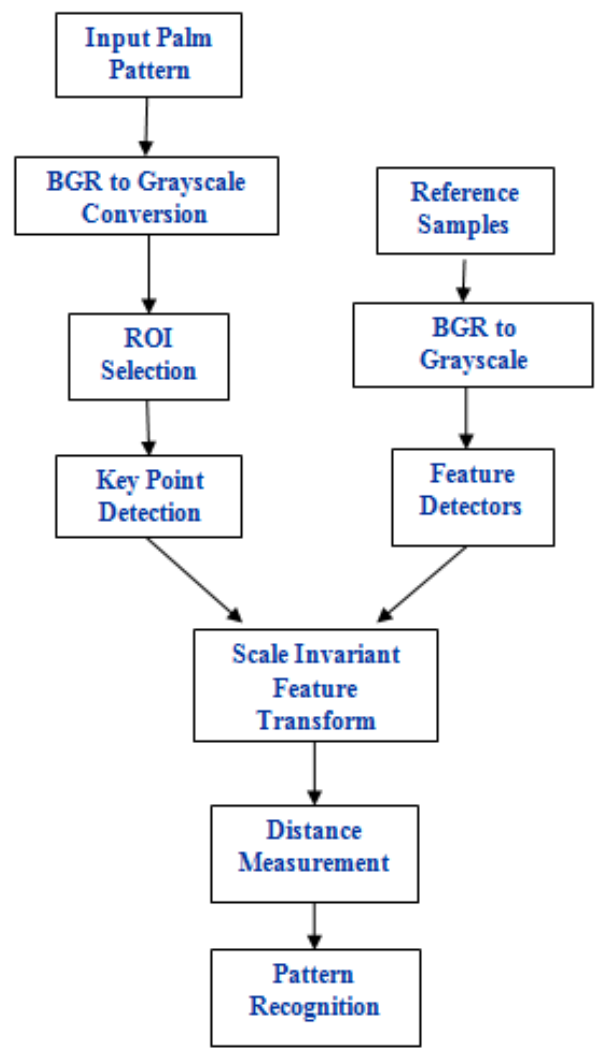

\subsection{Key point detection}

Key points are interest points that are locations of spatial or points used to give the changes in images such as image rotation, strings, or distortion. Scale and orientation play an important role in key point detection. The position and coverage area information of the image can be obtained after key point extraction. The key points that are detected on target palm pattern image which are matched based on distance measurement in target. Number of key points can be increased or decreased based on the image for our convenience. For example, Figure 2 shows the detection of key points for hand shape.

\subsection{Image matching using key point measurement}

Images consist of arrays of rows and columns based on pixels. Key points are used share a location of rows and columns. The target palm pattern image can be detected based on key point measurement and compared with reference palm pattern image using structural similarity (SSIM). Then matched key points on a detected image and reference is 
identified by drawing lines between the key points. The matching percentage accuracy can be calculated based on number of matching key point lines. For example, in Figure 3, it shows the key point matching based on distance measurement. It compares the texture of the palm pattern matching using key point measurement between target palm pattern image and reference palm pattern image.

Figure 2 Key points detection (see online version for colours)

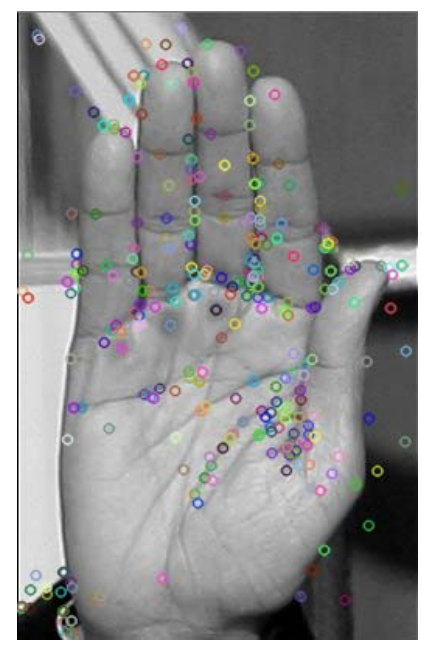

Figure 3 Key point matching based on distance measurement (see online version for colours)

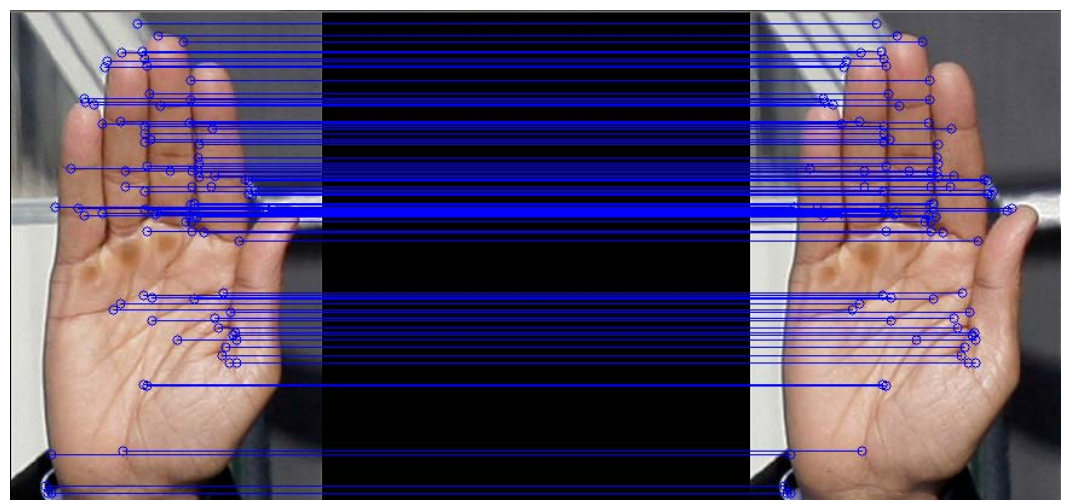

\section{SIFT algorithm}

SIFT algorithm is invariant to rotation, scale, brightness change. SIFT feature can be used to compute massive feature data and to combine with other types of feature matching. Palm patterns are composition of blood that is present in the skin. It can be obtained using infra red rays which protects against external damages, impersonation and spoof attacks. Contactless palm pattern has some advantages such as hygiene, non contact acquisition. In our proposed method, palm pattern segmentation is done using SIFT 
algorithm. SIFT algorithm is used for image matching and recognition and is invariant to translations, rotations and scaling transformations in the image. SIFT is used to estimate the scale space extrema using the difference of Gaussian (DOG) then used to find the key point localisation for eliminating the low contrast points. Finally, a key point orientation assignment has been done based on local image gradient. Then we find the image descriptor for each key point using image gradient magnitude and orientation. The SIFT algorithm is follows as,

- obtaining the target image

- computation of the scale space extrema of image using DOG function

- $\quad$ find the key point localisation

- computation of image descriptor.

The estimation of local extrema of each key point $(x, y)$ is given by

$$
\begin{aligned}
& D(x, y, \sigma)=(G(x, y, \sigma)-G(x, y, \sigma)) * I(x, y) \\
& D(x, y, \sigma)=L(x, y, s \sigma)-L(x, y, \sigma)
\end{aligned}
$$

where

$$
\begin{aligned}
& L(x, y, \sigma)=G(x, y, \sigma)^{*} I(x, y) \\
& G(x, y, \sigma)=\frac{1}{2 \pi \sigma^{2}} e^{-\left(x^{2}+y^{2}\right) / 2}
\end{aligned}
$$

where $\sigma$ is a scaling parameter and $L(x, y, \sigma)$ represents Gaussian Smoothed image at the key point with $\sigma$. The Taylor series expansion of the DOG scale-space function $D(x, y, \sigma)$ is used to find the interpolation for each key point is given by,

$$
D(x)=D+\frac{\lambda D^{T}}{\lambda x} x+\frac{1}{2} x^{T} \frac{\lambda^{2} D}{\lambda x^{2}} x
$$

The gradient magnitude $m(x, y)$ of an image and orientation $\theta(x, y)$ can be computed as follows.

$$
\begin{aligned}
& m(x, y)=\sqrt{(L(x+1, y)-L(x-1, y))^{2}+(L(x, y+1)-L(x, y-1))} \\
& \theta(x, y)=\tan ^{-1}\left(\frac{L(x, y+1)-L(x, y-1)}{L(x+1, y)-L(x-1, y)}\right)
\end{aligned}
$$

\section{Results and discussion}

The performance of the proposed SIFT method is based on feature detector and conventional method is discussed in this section. Using SIFT method, contactless palm pattern images can be acquired, matched, recognised, authenticated and their matching performance are simulated using OpenCV. OpenCV consists of many libraries of programming functions and its primary interface is python. The performance analysis and comparison of the SIFT transform and ORB are done using OpenCV-Python. We tested 
the method with a set of different texture of the palm pattern of different real time images nearly 20 images. The images are compared using SIFT and ORB algorithm based on key point matching. Figure 4 shows the simulation results of palm pattern matching using SIFT and ORB methods. Experimental results using the proposed method show that the SIFT method can produce detect face with high detection rate.

From Figure 4, the simulation results show that SIFT algorithm provides better image matching compared to ORB algorithm. SIFT recognises the palm pattern matching effectively for real time images. It produces high accuracy even when more key points are taken and gives superior results than ORB even with moisture and oil surfaces.

\section{Conclusions}

The finger prints authentication method leads to be fraudulent because it could be extracted easily from individuals. SIFT algorithm based on feature detection is proposed which is focused on contactless palm pattern images. Using SIFT method palm pattern images can be acquired, matched, recognised, authenticated and their matching performance are simulated using OpenCV. The simulation results demonstrated that SIFT algorithm achieves greater performance than ORB method on contactless palm pattern images that is suitable for real world scenarios.

Figure 4 Comparison of palm pattern images using SIFT and ORB algorithms based on key point matching (see online version for colours)

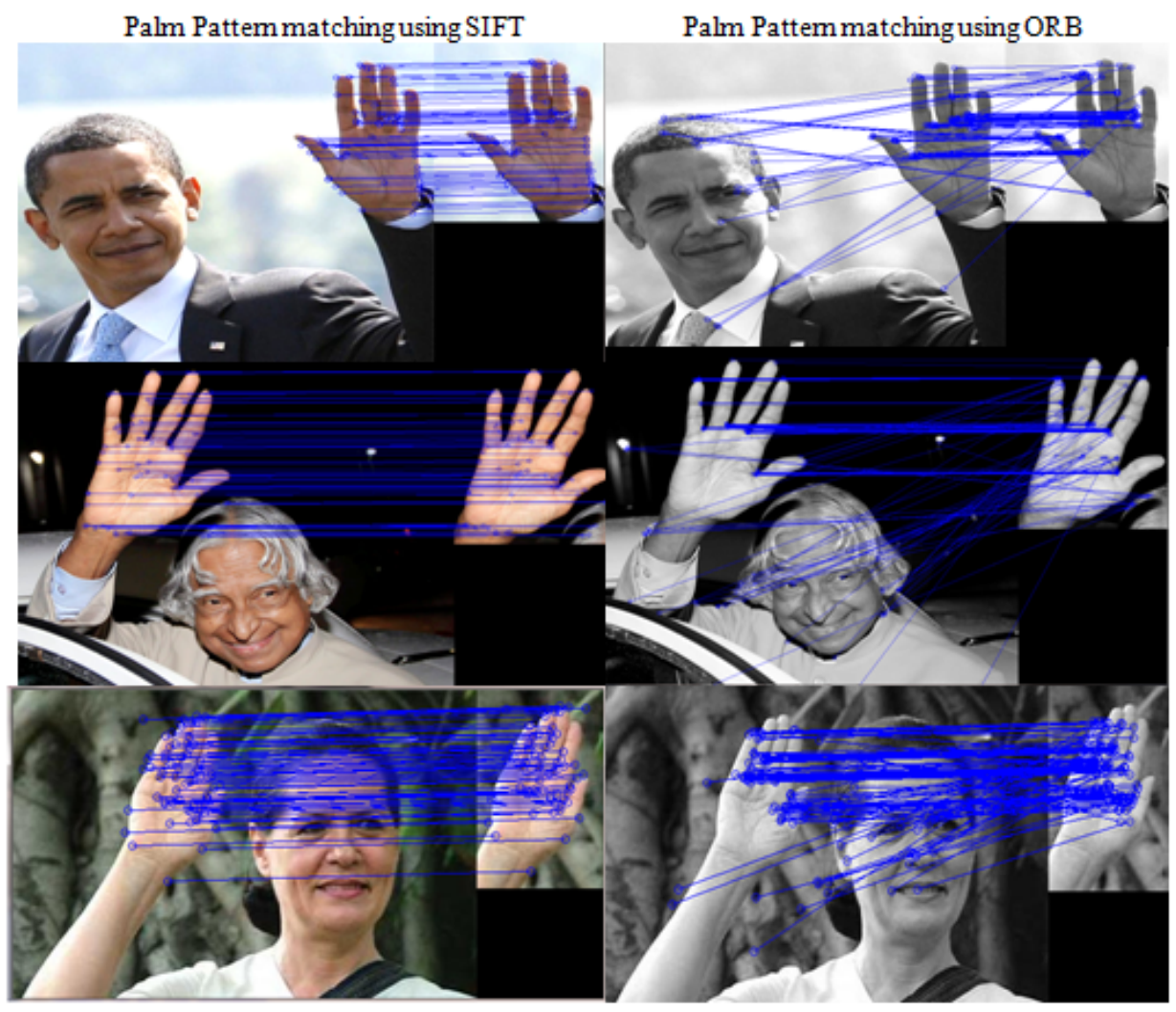




\section{References}

Bay, H., Tuytelaars, T. and Gool, L.V. (2008) 'Speeded-up robust features (SURF)', Computer Vision and Image Understanding, Vol. 110, No. 3, pp.346-359.

Chiu, L.C., Chang, T.S., Chen, J.Y. and Chang, N. (2013) 'Fast SIFT design for real-time visual feature extraction', IEEE Transactions on Image Processing, Vol. 22, No. 8, pp.3158-3167.

Fischer, M., Rybnicek, M. and Tjoa, S. (2012) 'A novel palm vein recognition approach based on enhanced local gabor binary patterns histogram sequence', 19th International Conference on Systems, Signals, and Image Processing (IWSSIP), Austria.

Gaikwad, D.P. and Narote, S.P. (2014) 'Multi-modal biometric system using palm print and palm vein features', Annual IEEE India Conference (INDICON), DOI: 10.1109/ INDCON.2013.6726010.

Güzel, M. (2015) 'A hybrid feature extractor using fast hessian detector and SIFT', Technologies, Vol. 3, No. 2, pp.103-110.

Huang, W., Wu, L. and Song, H. (2013) 'RBRIEF: a robust descriptor based on random binary comparisons', IET Computer Vision, Vol. 7, No. 1, pp.29-35.

Jia, L., Weiping, F., Wen, W. and Na, L. (2013) 'Image matching based on improved SIFT algorithm', Chinese Journal of Scientific Instrument, Vol. 34, No. 5, pp.1107-1112.

Jinbo, D., Hongwei, Z., Junlin, L. and Jia, F. (2012) 'A simplified SIFT algorithm for descriptor', Chinese Journal of Scientific Instrument, Vol. 33, No. 10, pp.2255-2262.

Joardar, S., Chatterjee, A. and Rakshit, A. (2016) 'Real-time NIR imaging of palm dorsa subcutaneous vein pattern based biometrics: an SRC based approach', IEEE Instrumentation \& Measurement Magazine, Vol. 19, No. 2, pp.13-19.

Karami, E., Prasad, S. and Shehata, M. (2015) 'Image matching using SIFT, SURF, BRIEF, and ORB: performance comparison for distorted images', Proceedings of the 2015 Newfoundland Electrical and Computer Engineering Conference, Canada.

Ke, Y. and Sukthankar, R. (2004) 'PCA-SIFT: a more distinctive representation for local image descriptors', IEEE Computer Society Conference on Computer Vision and Pattern Recognition, Vol. 2, pp.506-513.

Kumar, A. and $\mathrm{Xu}, \mathrm{Z}$. (2016) 'Personal identification using minor knuckle patterns from palm dorsal surface', IEEE Transaction on Information Forensics and Security, Vol. 11, No. 10, pp.2338-2348.

Liao, B. and Wang, H. (2015) 'The optimization of SIFT feature matching algorithm on face recognition based on BP neural network', Applied Mechanics and Materials, Vol. 743, pp.359-364.

Luan, Z. and Dalong, G. (2012) 'A SIFT feature descriptor based on fan-shaped region segmentation', Acta Automatica Sinica, Vol. 38, No. 9, pp.1513-1519.

Mahesh and Subramanyan, M.V. (2012) 'Automatic feature based image registration using SIFT algorithm', International Conference on Computing, Communication and Networking Technologies (ICCCNT'12), IEEE-20180, DOI: 10.1109/ICCCNT.2012.6396024.

Michael, G.K.O., Connie, T. and Jin, A.T.B. (2010) 'Design and implementation of a contactless palm print and palm vein sensor', 11th International Conference on Control, Automation, Robotics and Vision, Singapore, pp.1268-1273.

Moghaddam, B., Nastar, C. and Pentland, A. (2001) 'A Bayesian similarity measure for deformable image matching', Image and Vision Computing, Vol. 19, No. 5, pp.235-244.

Piciucco, E., Maiorana, E. and Campisi, P. (2018) 'Palm vein recognition using a high dynamic range approach', IET Biometrics, Vol. 7, No. 5, pp.439-446.

Shan, B. (2010) 'A novel image correlation matching approach', Journal of Multimedia, Vol. 5, No. 3, pp.268-275.

Shuzhen, Z., Hailong, S., Xiaoyan, X. and Yinnan, Z. (2010) 'Fast SIFT algorithm used in target recognition', Computer Systems and Application, Vol. 19, No. 6, pp.82-85. 
Srinithi, A. and Gopalakrishnan, B. (2017) 'Personal identification based on dorsal palm blood vessel pattern by texture feature', IEEE International Conference on Electrical, Instrumentation and Communication Engineering (ICEICE), DOI: 10.1109/ ICEICE.2017.8191952.

Vamsikrishna, K.M., Dogra, D.P. and Desarkar, M.S. (2016) 'Computer-vision-assisted palm rehabilitation with supervised learning', IEEE Transactions on Biomedical Engineering, Vol. 63, No. 5, pp.991-1001.

Wong, Y.W., Seng, K.P. and Ang, L.M. (2011) 'Radial basis function neural network with incremental learning for face recognition', IEEE Transactions on Systems, Man, And Cybernetics-Part B: Cybernetics, Vol. 41, No. 4, pp.940-949. 